\title{
OCCLUSAL SOUND ANALYSIS REVISITED
}

\author{
H.N. Teodorescu \\ Technical University of Iasi and Romanian Academy - Computer Science Institute, Iasi, Romania, e-mail \\ hteodor@etc.tuiasi.ro
}

Keywords: occlusal sound, gnathosony, statistical analysis, nonlinear analysis, Internet-based test

\begin{abstract}
We present a review of the gnathosonics technique, several improvements and a revision of concepts. Then, we present a method of nonlinear signal processing and heuristic features extraction. We also propose an adaptation of the technique to acquire the gnathosonics signal over the Internet.
\end{abstract}

\section{Introduction}

The gnathosonic analysis refers to the analysis of sounds produced during occlusion, that is, throughout the closing of the mandible over the maxillary at some stage in masticatorylike movements. Watt has initiated the analysis of these sounds with application to diagnosis of the state of the stomato-gnathic apparatus during the 1960s and 1970s. While the method has seen some interest [1-11], it has not established itself as a current method in clinical practice. There are several reasons for that. In the first place, the lack of a well established methodology in acquiring the occlusal sounds. Second, that happened because of the great intrasubject and inter-subject variability of the occlusal sounds. Several papers tied to refute or re-establish the credibility of the method based on the deterministic understanding of the occlusal sound. It has not been recognized that occlusal sounds represent a stochastic process and consequently the authors have not been able to provide a sound foundation of the gnathosony. Third, there is a lack of equipments to help establish gnathosony as a practical means. The only reported portable device for gnathosony, using air sound transmission, has been described in [10]. Throughout this paper we refer to the occlusal sound air transmitted, in contrast to the bone transmitted vibrations due to occlusion, typically analyzed in other papers.

\section{Occlusal sounds generation and sound features systematics}

The difference between a normal occlusion and the two main classes of static (final) malocclusions are illustrated in Fig. 1, where the positions of the teeth are shown. However, the occlusal sound is not relevant for the final, static type of occlusion, but for the dynamic process in the terminal phase of the occlusion, namely on the stability of the occlusion. Either a normal static occlusion or a malocclusion can have a correct stability in the final phase of the occlusion process, and any type of occlusion can show instability in that phase.
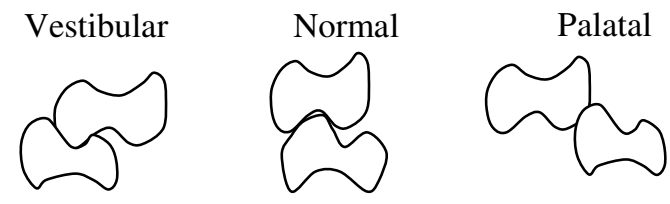

Fig. 1. Sketch of the normal occlusion and two typical malocclusions

The medical importance of the contact phase of the occlusion consists in that that multiple contacts and sliding produce the abrasion of the tooth and possibly trauma of the periodontium. Hence the importance of analysing the occlusal sounds and of developing a reliable procedure to determine the dynamic characteristics of the occlusion, based on these sounds.

A limit in the occlusal sound analysis has been the complexity and the variability of shapes of the sound wave. It has been proposed to analyze the occlusal waves after extracting the envelopes [10]. There is no thorough analysis and classification of the wave forms, or of the envelopes in the literature, and this might have been a reason why the field of gnathosonics ahs not evolved according to the initial expectations.

The shape of the envelope of an occlusal sound is determined by the number of occlusal contacts and by the dynamics of the terminal part of the occlusion, namely by the dynamics of the sliding of the teeth, from the first contact until the equilibrium position in occlusion. A characterization of the waveform should take into account the need to correlate the sound with the medically relevant processes of contact and sliding. Typical shapes of the envelopes of the occlusal sounds are shown in Fig. 2. The number of contacts, the duration of the overall sound, and the character of grinding noise are important.

The ideal sound corresponds to a single brief contact sound, with a single and well defined maximum (simultaneous occlusal contact), like in Fig. 2a. The decay section of the envelope can be assumed to be exponential [5]. Double occlusal contact produces a sound with twined maxima (Fig. $2 \mathrm{~b}$ ), when the contacts are produced almost simultaneously. The sound with the envelope sketched in Fig. 2c) corresponds to a double contact, with contacts occurring at a large time interval.

Two other cases of envelopes of occlusion sound are shown in Fig. 3. While they are not new truly classes, resembling to those in Fig. 2 b and 2c, they have some specific traits: distinct contact sounds and plateau. 
a)

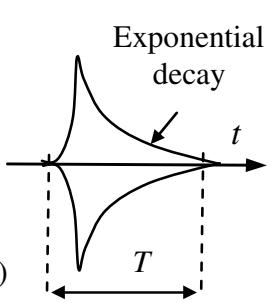

First occlusal contact

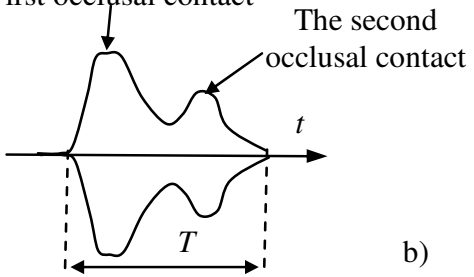

First occlusal contact

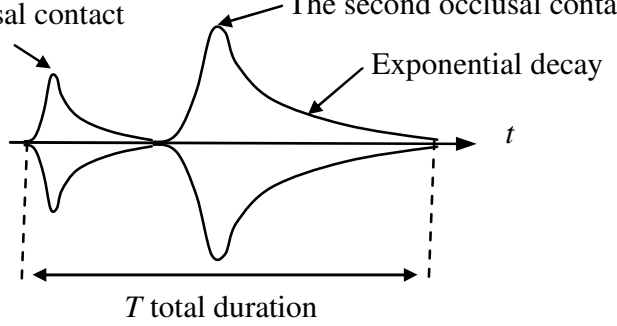

Fig. 2. Typical envelopes of the occlusal sounds
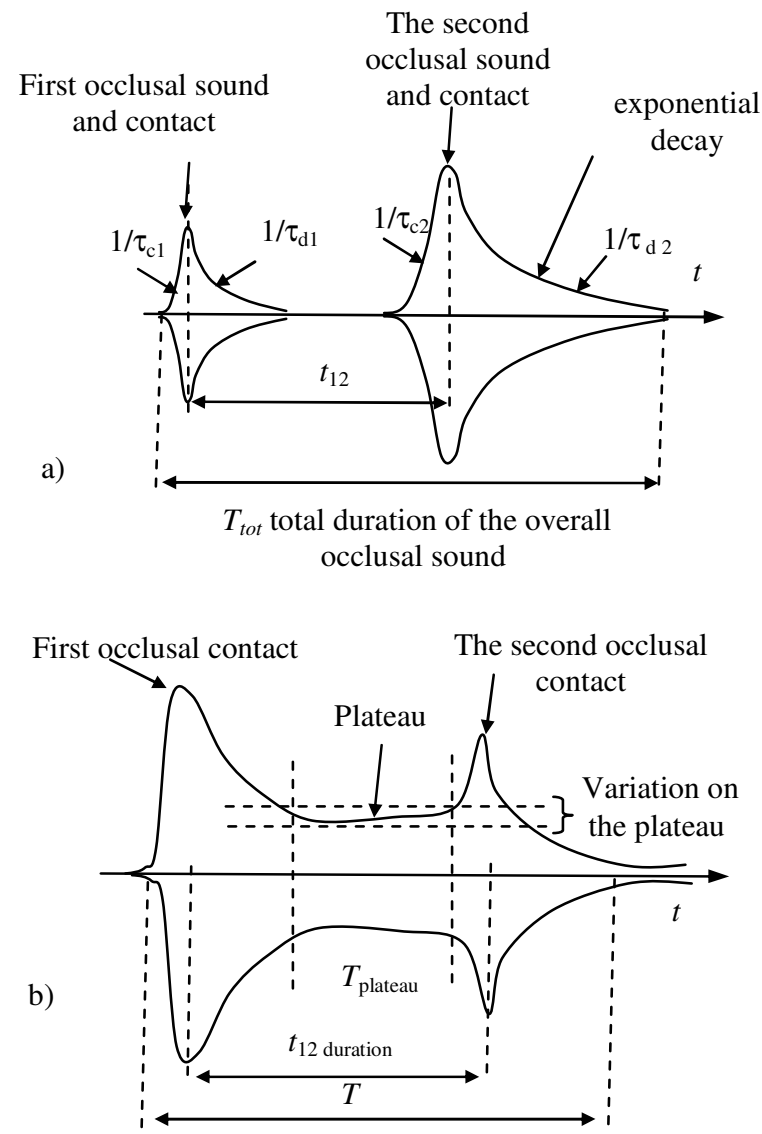

Fig. 3. Other possible cases of occlusion sounds: a) sound with two distinct maxima; b) sound with

plateau and the parameters definition

The envelope of a single contact sound is characterized by the rise and fall times, value of the maximum, duration of the maximum, and total duration. The rise and fall curves follow exponentially laws (see Fig. 4), whose constants are of interest in the classification of the occlusal dynamics. The automatic estimation of the parameters in Figures 2, 3 and 4 is an aim of this research.

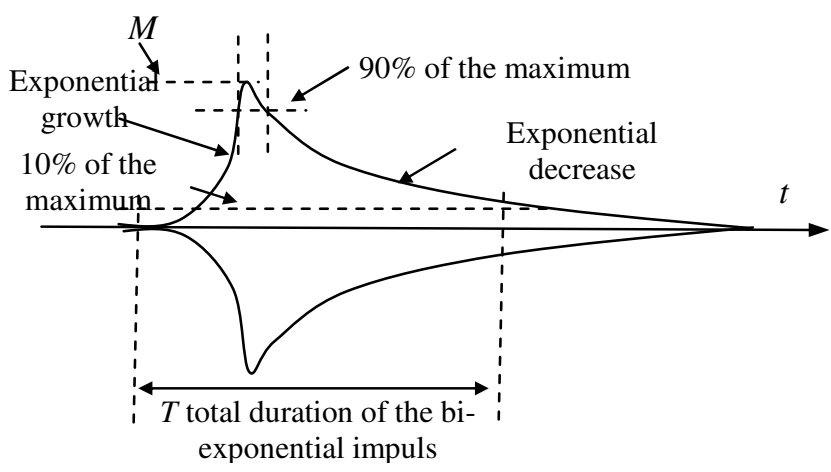

Fig. 4. Definition of the rise and decay time for a typical occlusal sound

Examples of acquired occlusion sounds that illustrate the above cases are shown in Fig. 5, 6 and 7. All the sounds have been acquired on a PC using a microphone. We preferred this method because it offers the potential of widespread homebased screening, as many subjects have home PCs on which they can perform the test. We have asked the subjects to naturally close the mouth as similar as possible to masticatory movements. The subjects have been asked to keep the lips parted in a natural way during the occlusion, for the occlusal sounds can freely travel to the microphone.

Fig. 5 represents a double contact occlusion, with the second contact more energetic, the two contacts being separated by a long time interval (about $10 \mathrm{~ms}$ ). In contrast, the occlusion in Fig. 6, while also exhibiting two contacts, the corresponding sounds are not separated by a pause. The first sound stands for a rather diffuse contact. The second maximum is "classic", with exponential rise and exponential decay.

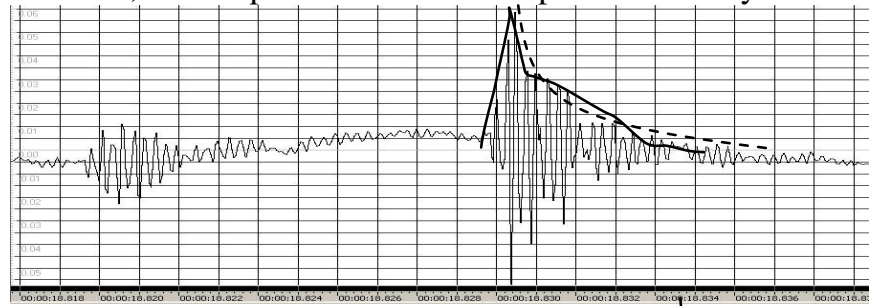

Fig. 5. Occlusal sound with two maxima separated by a long period of time. Approximate envelope and best approximating exponential decay sketched

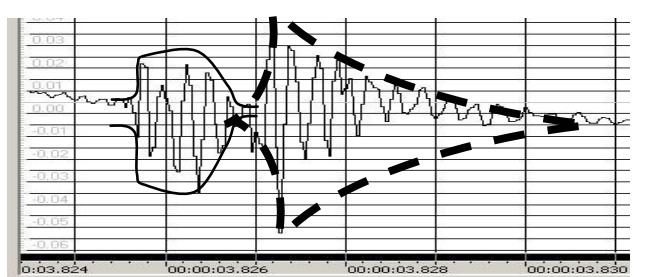

Fig. 6. Contact with two maxima that are close to each other yet well disjoint in time

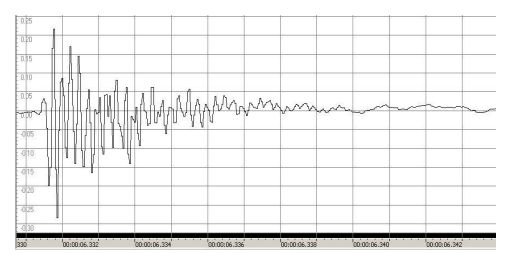

Fig. 7. Impact almost instantaneous. Increase time $<1 \mathrm{~ms}$ 


\section{Algorithm for envelope extraction}

The first step in occlusal sound features generation is the envelop extraction. In this purpose, we have used a non-linear filtering based on a mixture of maximum and minimum generation of the signal, and a modified, double extraction of the median, followed by low-pass filtering. The algorithm extracts the median on a window of four times the period of the "pitch", which is about $1 \mathrm{~ms}$. The low-pass filtering smoothes further the resulted waveform.

Denoting the signal by $s(t)$, the processing follows the steps:

$$
\begin{gathered}
u_{\text {inf }}[n]=\min _{k=-3 \ldots+3} s[n+k] ; u_{\text {sup }}[n]=\max _{k=-3 \ldots+3} s[n+k] \\
v_{\text {inf }}[n]=\frac{1}{7} \cdot \sum_{k=-3}^{3} u_{\text {inf }}[n+k], v_{\text {sup }}[n]=\frac{1}{7} \cdot \sum_{k=-3}^{3} u_{\text {sup }}[n+k] \\
z_{\text {inf }}[n]=\operatorname{med}_{k=-3 \ldots+3}\left\{v_{\text {inf }}[n+k\}\right\}, z_{\text {sup }}[n]=\operatorname{med}_{k=-3 \ldots+3}\left\{v_{\text {sup }}[n+k\}\right\} \\
y[n]=\left(z_{\text {inf }}[n]+z_{\text {sup }}[n]\right) / 2 \\
x[n]=(y[n-p]+y[n-p+1]+\cdots+y[n]) /(p+1)
\end{gathered}
$$

where $p+1$ is the order of the averaging filter. We used with good results a filter with $p=5$. Examples of noisy recording and of its pre-processing are shown in Figs. 8 and 9.

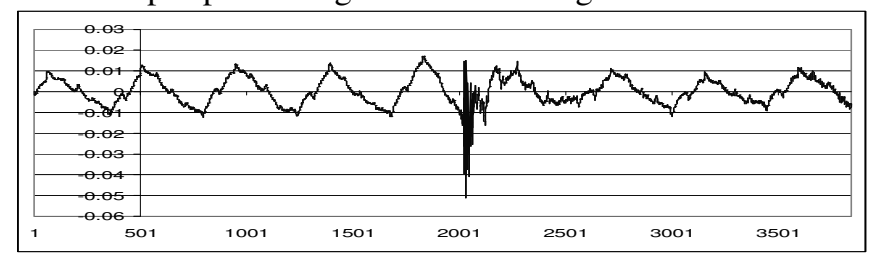

Fig. 8. Example of noisy initial record

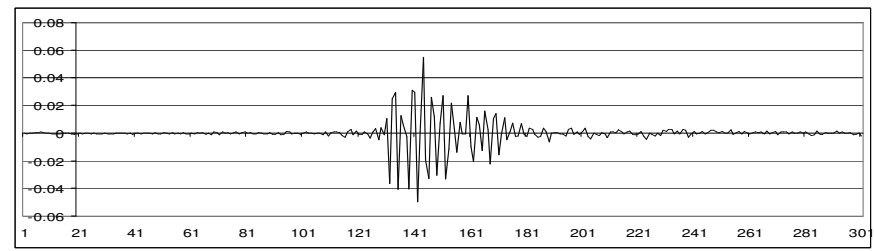

Fig. 9. After pre-processing for noise removal of the signal in the previous figure

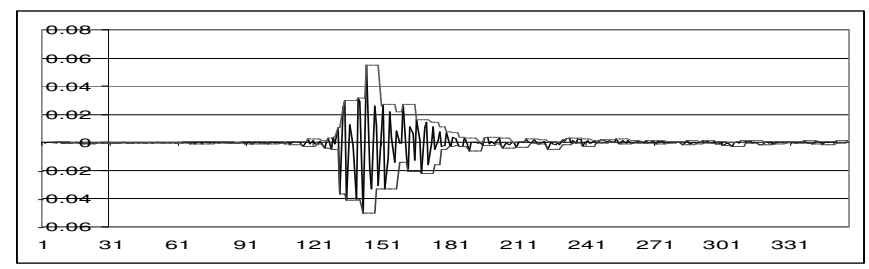

Fig. 10. After envelopes extraction

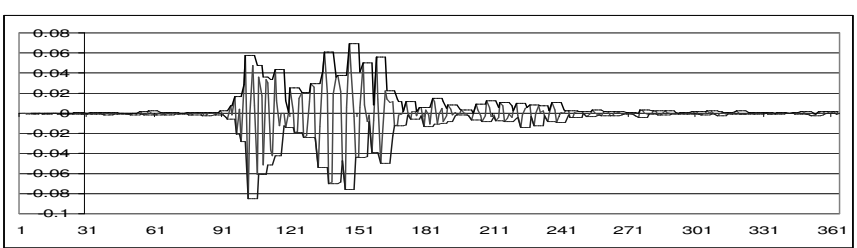

Fig. 11. Envelope of a two-contact occlusion sound

The resulted waveform is analyzed with respect to the number of maxima, plateaus, and rise and decay times, as shown in Figs. 10-12.

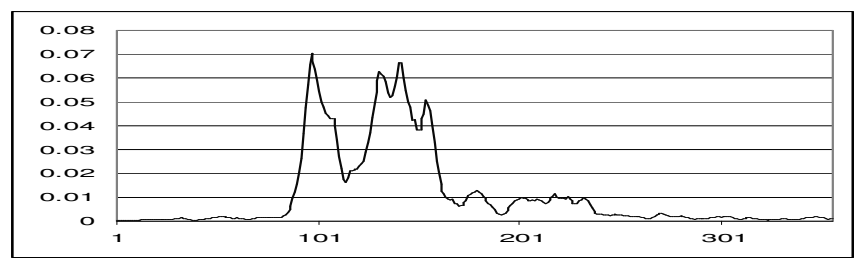

Fig. 12. Envelope after smoothing

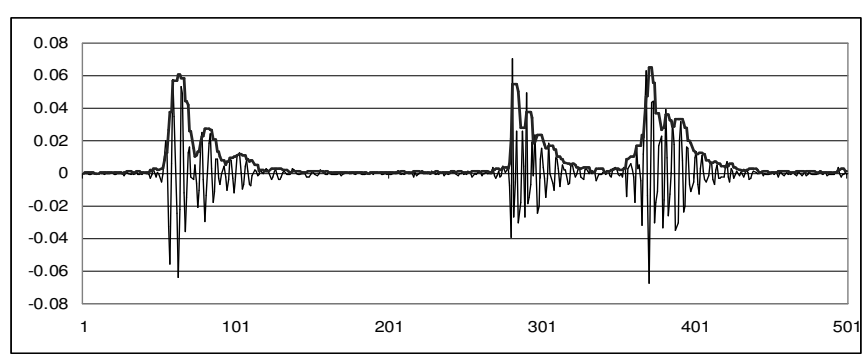

Fig. 13. A triple contact sound with the overall envelope detected

\section{Features extraction}

A heuristic method for features extraction is used, based on mimicking the human visual approach of visual interpretation of the occlusal waveform. First, the duration of the pulses and of the number of pulses are estimated. The pulse is naively defined by the time of the level higher than a threshold. However, with this definition, large errors are possible. More conditions have to be imposed, including the fact that the previous samples have large enough values and that subsequent values are also high. With these conditions added, small "gaps" in the signal will not produce false impulses. In Fig. 14a), false impulses are generated when applying the naïve method. The proposed algorithm produces accurate detection of the impulses, like in Fig. 14b).
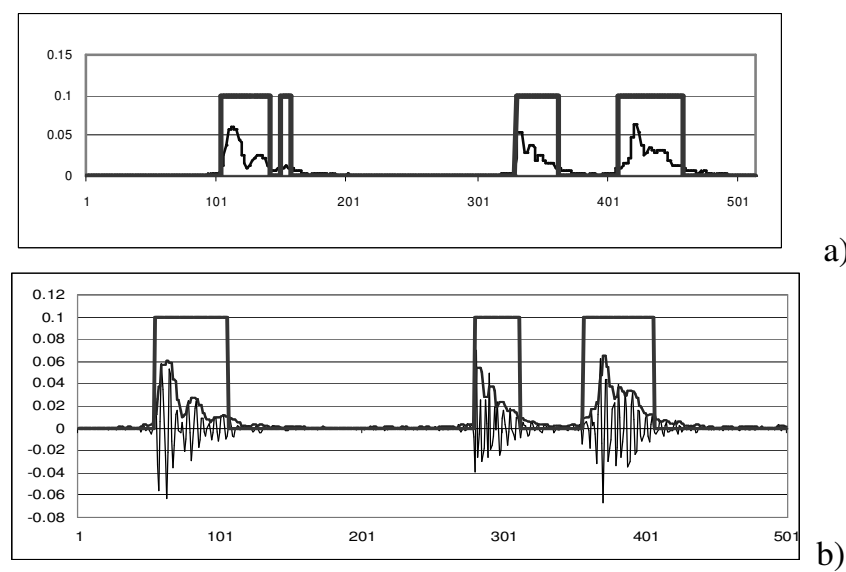

Fig. 14. Extraction of the number of contacts and of the duration. Notice the wrong result yielded by the threshold-based algorithm (a)

The identification of every independent contact sound, the determination of individual absolute maxima of the contact sounds and of the corresponding rise time is also performed by an heuristic procedure, to correspond to the medical expert interpretation. An example of the results is shown in Fig. 15. 


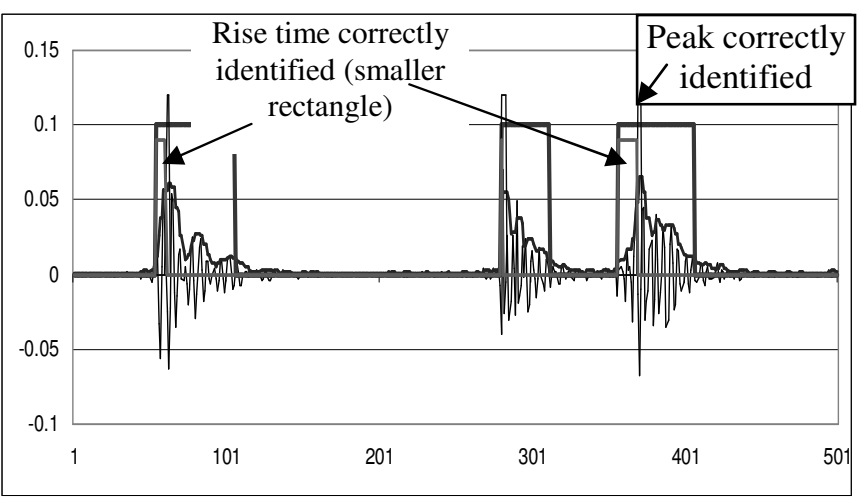

Fig. 15. Determination of the absolute maxima of every contact sound and of the rise time.

\section{Statistical processing - preliminary findings}

While the object of this paper is to present a method to automatically analyze the occlusal sounds, not the discussion of the medical benefits of occlusal sound analysis, preliminary information derived in this research may deserve mentioning. There is a strong inter- and intra-variability of the occlusal sounds. However, we found that the averages of the main parameters, moreover the variances of the parameters are informative in assessing the contact phase of the occlusal dynamics. That is, while parameters of individual occlusal sounds may be misleading, statistical parameters are reproducible and significant. Therefore, we performed a statistical analysis of the occlusal sounds, based on at least ten recordings per session, obtained from several subjects. Several sessions have been arranged for several subjects, with several days between the sessions. Both healthy subjects and subjects with troubles of the occlusal dynamics have been analyzed. We found that the statistical parameters are reproducible between sessions. Moreover, we found that statistical parameters can reveal several troubles in the dynamic malocclusions, including neurological, articulatory and motor troubles.

\section{Further development: Internet operation}

The proposed Internet operation consists in using the voice feature of one of the voice messengers, like Yahoo Messenger ${ }^{\mathrm{TM}}$. Such tools allow the transmission of acoustic signals in a form suitable for recovering at the other end and for analyzing the occlusal sounds transmitted by the correspondent. We started to explore this avenue and are currently building a screening tool for mass screening and prevention of occlusion-related troubles.

\section{Conclusions}

After systematizing the types and parameters of the occlusal sounds, we have presented a sketch of the method to acquire and analyze the air transmitted occlusal sounds. The occlusions are dealt with like statistical events. The statistical parameters are used to classify the sounds and to determine the type of dynamical malocclusion.
The processing method is robust enough to remove even high amplitude noises, when the noise is of industrial type (low frequencies); moreover, the method is robust enough to correctly identify the number of occlusal sounds, their duration and other vital parameters in the classification. A method of acquiring and analyzing the occlusal sounds via Internet has been also proposed for mass screening purposes.

\section{Acknowledgements}

The research for this paper has not been performed as part of any work with the funding of organizations. The results remain the property of the author. However, the work helped applying for a national grant (CEEX grant) and the participation in MEDISP2006 is funded by that grant. Thanks are due to all subjects who benevolently gave informed consent during several years of creation of the author's database of occlusal sounds.

\section{References}

[1]. Y. Asazuma, Y. Isogai, K. Watanabe, K. Hara, Changes in gnathosonic and tooth contact characteristics induced by experimental occlusal interferences created using a full-cast double crown. J Oral Rehabil. 1995 Mar; 22(3):203-11.

[2]. Freer TJ, Smith A., The reproducibility of gnathosonic recordings over time. Aust Dent J. 1989 Jun; 34(3):245-53

[3]. Fuller DJ, West VC., The tooth contact sound as an analogue of the "quality of occlusion". J Prosthet Dent. 1987 Feb; 57(2):236-43.

[4]. Hedzelek W, Hornowski T., Gnathosonic study of occlusion in patients wearing complete dentures. Eur J Prosthodont Restor Dent. 1997 Sep; 5(3):119-23.

[5]. Hedzelek W, Hornowski T., The analysis of frequency of occlusal sounds in patients with periodontal diseases and gnathic dysfunction. J Oral Rehabil. 1998 Feb; 25(2):139-45.

[6]. Prinz JF., Computer aided gnathosonic analysis: distinguishing between single and multiple tooth impact sounds. J Oral Rehabil. 2000 Aug; 27(8):682-9.

[7]. Prinz J.F., Ng KW., Characterization of sounds emanating from the human temporomandibular joints. Arch Oral Biol. 1996 Jul; 41(7):631-9.

[8]. Shi CS, Mao Y., Elementary identification of a gnathosonic classification using an autoregressive model. J Oral Rehabil. 1993 Jul; 20(4):373-8.

[9]. Taguchi S, Sugita N, Asazuma Y, Hara K., Duration patterns of percussion sound in healthy and periodontally affected teeth. J Oral Rehabil. 1990 Nov; 17(6):579-85.

[10]. Teodorescu HN, Burlui V, Leca PD. Gnathosonic analyser. Med Biol Eng Comput. 1988 Jul; 26(4):428-31.

[11]. Tyson KW., Monitoring the state of the occlusion-gnathosonics can be reliable. J Oral Rehabil. 1998 May; 25(5):395-402. 